\title{
ON THE NEW PROSPECTS IN BIOLOGY INSPIRED BY EPIGENETICS
}

\author{
Maria E. Krasteva* \\ Laboratory of Genome Dynamics and Stability, Institute of Plant Physiology and Genetics, \\ Bulgarian Academy of Sciences, Sofia, Bulgaria
}

The epigenetic revolution that has happened very recently in biology has challenged the long-term traditional views of the genetic code. A new scientific era has emerged. These outstanding data made us reconsider major concepts in molecular genetics and outlined new perspectives, some of which are discussed here. First, it became apparent that heredity is determined not only by the primary DNA sequence but also by epigenetic factors controlling whether and when a gene will be turned on or off. These include modifications in the DNA or the associated proteins, which modulate gene function by causing changes in chromatin that affect DNA accessibility. Second, it turned out that though conservative, the genome is dynamic and changeable in response to environmental conditions. Third, a new value to nature vs. nurture debate was added demonstrating that these are not separate aspects but have a combined influence mediated through epigenetics. Forth, the epigenetics outburst contributed also to another controversial scientific field - the transgenerational inheritance. Evidence was provided that there are mechanisms for epigenetic transmission of environmentally induced changes that occur long before conception from a parent to the offspring. Fifth, the role of the psyche was given greater importance after the discovery that emotional stress may cause epigenetic changes in our heredity molecules. And sixth, a role of epigenetics in the disease was also established since epigenetic changes were related to many human disorders, thus providing possibilities for the development of more effective therapeutic approaches. Biomed Rev 2020; 31: 31-39

Keywords: Epigenetics, Environment, Transgenerational epigenetic inheritance, Psyche, Disease

\section{INTRODUCTION}

The characterization of the molecular structure of DNA and its function as the unit of heredity heralded the field of molecular genetics. This has become the leading and one of the most promising fields in modern science. The hereditary molecule of living organisms was attributed too much expectation in view of achieving almost every possible aspiration in science. But, as it often happens, when we are so focused on something, it strongly distorts the real prospect of it. And I guess, that was what happened with DNA.

*Correspondence to: Dr Maria E. Krasteva, Laboratory of Genome Dynamics and Stability, Institute of Plant Physiology and Genetics, Bulgarian Academy of Sciences, Acad. G. Bonchev Street, Bldg. 21, BG-1113 Sofia, Bulgaria E-mail: maria_krasteva@abv.bg 
For more than half a century, this intriguing molecule was considered the mere determinant of almost all cellular functions. The DNA enthusiasm has reached its peak in the pursuit of a large-scale research known as Human Genome Project. A historical event happened in June 2000 when it was announced that the human genome has been sequenced. This discovery was by all means outstanding. However, the results were, to say the least, unexpected. Considering the size of the human genome ( $\sim 3$ billion bp), the anticipated number of genes was estimated to about 100000 . In fact, it turned out that the number of human genes is only about 20000 , and the coding sequences are just 1 to $2 \%$. Thus, about $98 \%$ of the human genome was first assigned the status of non-functional (1), conveying little or no selective advantage to the organisms, i.e. "junk" or "selfish" DNA (2). It is now known that a substantial fraction of the extra DNA has repetitive nature comprised of introns, pseudogenes, satellite sequences and transposable elements (TEs) interspersed with non-coding RNA genes. For example, TEs amount to more than $45 \%$ of the genome (3).

Why this apparently useless non-protein coding DNA has been still maintained by eukaryotic genomes? What is its function? Questions that puzzled scientists for a long time. The ENCODE project provided the first significant breakthrough into the understanding the proportion of human genome that is functional (ENCODE Project Consortium 2012). The discovery that the non-coding DNA plays important functions in genome integrity and functionality has challenged the concept of useless “junk" DNA. Some non-coding DNA are transcribed into functional non-coding RNA molecules, such as tRNAs, rRNAs, and regulatory RNAs engaged in processes such as the transcriptional and post-transcriptional epigenetic silencing, translational regulation, DNA replication, maintenance of the chromosome structure. Scientists started to pay more attention to TEs and their function in eukaryotic genomes including human. First of all, many TEs turned out to be functional. Their DNA is biochemically active, encode proteins, bind proteins, synthesize regulatory RNA, thus meeting the ENCODE criterion of functional elements. Second, many TEs are not "junk" as there is plenty of ways through which they provide a benefit to their host genomes in terms of genetic diversification and regulation of genome functioning.

Furthermore, it became apparent that when it comes to the genome sequences themselves, the organisms are very similar. For example, the differences between the genomes of the human and the chimpanzee are less than $2 \%$. Another observation is the lack of co-linearity between genome size, organism complexity and the number of genes that is in the core of the C-value paradox raised more than 30 years ago, where ' $C$ ' stands for the haploid genome size (4). As an example, humans, though much more complex than microscopic worms, contain a similar number of genes, indicating that the huge phenotypic differences between the two species are not a question of number. It is now demonstrated that the dynamics of the repetitive fraction of the eukaryotic genome, predominantly TEs, mainly contribute to this paradox. We began to understand that the level of genome and organism complexity comes exclusively from the level of the functional organization of a genome.

Overall, it turned out that the DNA sequence alone gives very incomplete knowledge of how the genome really functions and this put the focus on genome regulation and the control over gene expression. Studies showed that DNA is far from being a rigid molecule - on the contrary, it is changing relatively to changes in the environment. Thus, the beginning of $21^{\text {th }}$ century has marked the emergence of a new era in biology. The era of epigenetics. The long-term dogmatic views on heredity based exclusively on the genetic code inheritance were seriously questioned and new prospects have emerged. By challenging some traditional beliefs in biology, the postgenomic revolution has laid down questions, some of which are discussed below.

\section{HEREDITY IS DUE NOT ONLY TO PRIMARY DNA SEQUENCE}

The manifestation from genetic information to phenotype is dependent on epigenetic regulation. The description of epigenetics has evolved over time diverting significantly from Waddington's original definition (5) and refers to "the study of changes in gene function that are heritable and that do not entail a change in DNA sequence" (6). These are modifications in the DNA or the associated proteins, which modulate gene expression by causing changes in DNA-histones interactions responsible for chromatin remodeling into domains such as euchromatin, constitutive or facultative heterochromatin. The chromatin state affects the accessibility of the DNA molecule allowing it to undergo biological processes including transcription, replication or repair. It is not the genetic code itself that undergoes changes, but the way it is expressed in the specific cell type and organism. Epigenetic modifications arise independently of changes in the primary DNA sequence and can be transmitted to daughter cells.

"Epi" stands for "over" and "above" implying an additional 
level of hereditary information, on top of the genetic information, which allows differential regulation of a single gene expression depending on the surrounding circumstances. "On top of" could be referred not only to structure regarding the levels of chromatin organization, but also to function regarding the superior role of epigenetic mechanisms in the regulation of gene expression. While the genome provides the book of instructions, the epigenome adds further information of how those instructions should be completed to facilitate a particular functional outcome.

Three major groups of mechanisms for epigenetic regulation could be distinguished that can initiate and sustain epigenetic changes: methylation of DNA, post-translational histone modifications and non-coding RNAs. DNA methylation occurs at the $\mathrm{C}-5$ position of cytosine in CpGs often located at the promoters of genes. Cytosine methylation is a post-replicative process mediated by DNA methyltransferases and S-adenosyl-methionine being a donor of methyl groups. Methylation in DNA is responsible for the transcriptional silencing of the genetic material and plays an active role in development, $\mathrm{X}$ chromosome inactivation in females, and DNA imprinting. DNA methylation prevents the transcription of highly repeated genome sequences such as inserted viral sequences and transposons, which are potential sources of genetic instability. Histone modifications are covalent posttranslational modifications of histone proteins and include methylation on lysine or arginine residues, phosphorylation on serine or threonine, acetylation and deacetylation of lysines, and ubiquitylation and sumoylation of lysines. They are shown to be involved in transcription and chromatin remodelling processes. Regulatory short (siRNAs, miRNAs, and piRNAs) and long non-coding RNAs (lncRNAs) were also found to play a significant role in post-transcriptional epigenetic regulation. A predominant fraction of regulatory RNAs is synthesized from TEs. In addition, TEs are actually the main target of epigenetic mechanisms as they concentrate most epigenetic repressor marks in the genomes (7).

Epigenetic changes have at least a few unique features. First, they are much more frequent compared to genetic changes and usually arise in particular genome regions, often displaying cellular, tissue or organism-specific pattern. Second, epigenetic events do not affect the structure of the primary DNA sequence, and therefore, they are in principle reversible. Third, being mitotically inheritable they can have a long-lasting effect throughout the lifespan of an individual and can possibly be passed on to the next generation. And forth, as epigenetic events are unusually responsive to endogenous or exogenous factors, especially during critical stages of development, they could be sensitive barometers of organism-environment interactions.

\section{STABILITY THROUGH CHANGE}

It's not a question to debate on that the maintenance of the exact DNA sequence, which has been established through many years of evolution, is critical for normal cellular function and development. However, it turned out that though conservative, the genome is quite dynamic and changeable in response to the environment. The novel epigenetic data obliged us to admit that "a phenomenon can have a mechanistic basis, without being deterministic" (8) and "molecular" does not by rule mean "fixed".

Bioorganisms have developed sophisticated mechanisms of gene regulation. They respond to the challenging environment altering epigenetically. Many environmental factors were described to affect gene expression including temperature, diet, pollutants, allergens, drugs, infectious pathogens, heavy metals, and psychological stress. Several nutrient components, for example, were linked to alterations in DNA methylation or HDACs activity (9). Air pollutants were shown to cause methylation changes in blood DNA as reported for retrotransposon LINE-1 (10). A new field called environmental epigenetics was developed to study the interaction between the environment and the epigenome.

The early developmental stages are most vulnerable to stress responses mediated by epigenetic mechanisms. During embryogenesis, epigenetic marks undergo critical modifications due to the high level of cell division. Exposure to environmental stress during this period can cause epigenetic alterations, which may increase the risk of disease and dysfunction at later stages during lifespan (11). Let us take as an example studies on Dutch Famine Winter (1944-1945) survivors. Individuals prenatally exposed to famine displayed methylation changes in gene loci, involved in metabolic and cardiovascular disorders, which differed depending on the period of starvation and the sex (12). In addition, a decrease in DNA methylation of the imprinted insulin-like growth factor 2 (IGF2 ) gene was observed in exposed compared to unexposed siblings (13).

Another well-studied early life stressor, which may exert severe effects on the preconceptual and prenatal life, is the consumption of alcohol. Ethanol exposure during gestation leads to dysregulation in the structure and function of the embryonal nervous system. Exposure to mild levels of alcohol 
can result in a range of neurobehavioral phenotypes such as deficits in attention and perception, problems in executive functions, and hyperactivity. Chronic prenatal exposure to high alcohol doses is associated with a spectrum of clinical manifestations called Fetal Alcohol Syndrome (FAS) (14). Alcohol intake is involved in the modulation of the cellular immune response and the increase in infections susceptibility (15). A large body of evidence has shown that at least some of the alcohol effects are maintained through epigenetic processes, mainly DNA methylation and histone modifications $(16,17)$. Prenatal exposure to ethanol was shown to affect the neurotrophins signaling pathways causing a disruption in NGF and BDNF levels (18). Similar to maternal, paternal alcohol consumption alters NGF and BDNF expression in the mouse brain and intensifies the rewarding effects sensitivity upon ethanol administration in the male offspring (19).

Another example of DNA sequence invariability, which seems not to be aimed by all means, comes from the study of such processes like DNA replication and repair, which until recently have been ultimately considered conservative. It was shown that in a stressful environment some microorganisms use DNA polymerases able to tolerate DNA replication errors (20). So, is it possible that upon high stress the organisms may react in a way to enable a mutational event? If so, these will generate a sort of genetic diversity for the emergence of new variations, at least some of which may turn to be advantageous. Can we propose that in life-threatening conditions, it may be most important for the organism to survive without taking into account the possible long-term consequences?

Moreover, reversible changes have been reported to affect genetic integrity as part of the normal cellular functions (21). A control over DNA damage and repair has even been proposed. So, is it possible that the multicellular organisms may "purposely" generate promoter damage to enable normal gene regulation?

\section{IT'S NOT NATURE OR NURTURE, IT'S BOTH}

Epigenetics has added a new value in nature nurture debate, one of the oldest issues in science, which focuses on the relative impact of nature (genetic inheritance) and nurture (environmental factors) for the development. For a long time, the genes and the environment were considered separate units interacting only on the phenotypic level. However, it turned out they are closely related and their combined impact is mediated through epigenetics.

The impact of the environment on the heredity can be bril- liantly illustrated by studies on monozygotic twins. As they originate from one and the same zygote, their embryos are genetically identical. However, the environment may affect in a totally different manner each of the twins resulting in a totally different outcome. The differences may not only be related to the manifestation of certain phenotypic characteristics, but also to the development of specific diseases triggered by epigenetic changes. Diseases resulting from a specific genetic mutation, like for example achondroplasia (a form of dwarfism), show a $100 \%$ concordance rate. However, the majority of human disorders are not caused by a single mutation in a key gene but rather have a much more complicated nature. Let us take schizophrenia as an example. Schizophrenia affects from 0.5 to $1 \%$ of the population and its concordance rates for nonidentical and identical twins are $17 \%$ and $50 \%$, respectively. Though this is indicative of the existence of a major genetic component predisposing to schizophrenia, if one monozygotic twin develops the disease, there is still a $50 \%$ chance for the other not to get ill. This depends exclusively on the environment. A substantial genetic burden was found also for a number of other human diseases including bipolar disorder, multiple sclerosis, asthma and others.

Furthermore, genetically identical individuals become epigenetically different with age. A team headed by Jeffrey Craig investigated the DNA methylation pattern in newborn twin pairs at the Royal Children's Hospital in Melbourne and found that monozygotic twins begin to diverge epigenetically even during their intrauterine development (22). The observed differences became more pronounced over time as a consequence of the influence of the environment.

Perhaps the most exciting deduction that arose from epigenetics was that we are not doomed by our DNA. A revolutionary study of Waterland and Jirtle demonstrated that the nutrient diet may silence the effect of a specific mutation without altering the gene sequence (23). The authors used a model system of viable yellow agouti $\left(\mathrm{A}^{\mathrm{vy}}\right)$ mice, which harbor a retrotransposon in the gene. They found that dietary methyl donor supplementation of a/a dams alter $\mathrm{A}^{\mathrm{vy}} / \mathrm{a}$ offspring phenotype by increasing $\mathrm{CpG}$ methylation in the retrotransposon at the locus. The comprehension that such a minor change in the nutrient diet of the mother during her pregnancy could have such a dramatic impact on the offspring phenotype gave us responsibilities for which we were hardly prepared. Another remarkable data of the team led by Adrian Bird demonstrated that even neurological conditions, such as the Rett syndrome, can be reverted to normal through 
the mediation of epigenetics (24). These discoveries somehow broke the double-stranded handcuffs of our DNA and quite unexpectedly we faced the perspective of obtaining a certain level of control over our genes.

\section{FROM "THE WISDOM OF THE BODY" * TO THE WISDOM OF THE MOLECULES}

The outburst in epigenetics has contributed also to another controversial scientific field, the transgenerational inheritance. As discussed above, epigenetic changes can occur during the lifespan of an individual as a result of the interaction with the environment. Such events could even happen during the development of the embryo in the womb. However, are there similar mechanisms for the transmission of environmentally induced epigenetic changes from a parent to the offspring, which occurred long before the conception?

Currently, the use of the term heritability in the context of epigenetics is usually restricted to inheritance through mitotic events. However, though the evidence of transgenerational epigenetic inheritance is not consistent across organisms, several examples could be pointed.

Dias and Ressler (25) studied how the olfactory experience of a parent before conception may influence the behavior and epigenetics of the offspring. By using specific odors as conditioned stimuli the authors examined the inheritance of parental traumatic exposure. They subjected F0 mice to odor (acetophenone) fear conditioning before conception and found that F1 and F2 generations showed an increased behavioral sensitivity to the F0-conditioned odor but not to other odors. Therefore, the offspring somehow had inherited the information concerning the specific stimuli present in the parental environment in such a way to allow for appropriate stimulusspecific responses. The authors demonstrated that such an inheritance is epigenetically mediated as bisulfite sequence analysis revealed methylation differences in $\mathrm{F} 0$ and $\mathrm{F} 1$ generations that may be responsive for the enhanced transcription of specific olfactory receptor genes in the subsequent generations. Dunn and Bale (26) demonstrated epigenetic inheritance of body length and glucose homeostasis (GH) depending on the maternal diet. The authors reported a significant body length increase and reduced insulin sensitivity across at least two offspring generations in response to high-fat maternal diet exposure. Growth-promoting alterations in the GH pathway

\footnotetext{
* The Wisdom Of The Body by Walter B. Cannon was published in 1932 by NY: W.W. Norton.
}

were observed. A study in rats demonstrated that a single exposure of the ancestors to the environmental compound vinclozolin modifies the descendants' response to this stress factor in the third generation altering neural genomic activity that correlates with changes in physiology and behavior (27).

In addition, some of the effects of the Dutch famine on survivors were observed in the next two generations. Children who were exposed to intrauterine undernourishment and their offspring developed obesity, glucose intolerance and coronary heart disease later as adults $(13,28,29)$. From an evolutionary perspective, the transgenically inherited flexibility in the body size could provide a mechanism for a rapid adaptation of the organism. So, could the appropriate modification of the body size according to the change of food availability be advantageous? Is it possible that the dramatic environmental change, such as the decrease in food, may significantly cause adaptive epigenetic processes in the fetal cells leading to metabolic changes. If so, the biological meaning of these would be to allow the foetus to develop as healthily as possible regarding the stressful conditions (the decreased nutrient supply).

Can we suppose that the transgenerational epigenetic inheritance may provide a mechanism of learning from the ancestors' experience? If so, the transmission of beneficial survival solutions acquired by the parents during their lifetime will allow the offspring to exploit already verified responses in case a similar situation is encountered. The "wisdom" of the parents scripted in their DNA molecules may optimize progeny decisions to increase survival in a stressful environment.

A very recent study in C. elegans discovered that animals who have learned to avoid the pathogenic bacteria Pseudomonas aeruginosa (PA14) pass this learned behavior on to the next four generations. Piwi/PRG-1 is required for the transgenerational inheritance of PA14 avoidance. The offspring inherits PA14 avoidance based survival advantage, suggesting an adaptive response (30).

The above studies have shown that at least in some organisms new phenotypes can be generated and transmitted to the subsequent generations through epigenetic modifications. The transgenerational epigenetic learning could be evolutionarily beneficial. Perhaps, these may not be too significant on a large phylogenetic scale, but they seem significant enough on an organism scale, which spans throughout the lifespan of the individual and his next descendants.

\section{PSYCHE DOES REALLY MATTER}

We have always anticipated that emotional state is somehow 
related to physical health and lifespan. Nevertheless, the role of psyche has been more or less underestimated and the possible mechanisms underling this correlation - not well known. Yet, even in our most daring predictions, we could hardly imagine that emotions are able to affect heredity. Indeed, emotional stress was shown to cause epigenetic changes in our heredity molecules and that prospect was truly exciting.

Let us take early childhood abuse as an example. The effects of early traumatic experience have long-term consequences on the psyche and health. Children who have experienced adversity or neglect during childhood have increased blood cortisol levels as a result of compromised negative cortisol feedback to hypothalamic-pituitary-adrenal response to stress. A positive history of childhood abuse correlated with increased methylation in the GR promoter, which prevents the binding of NGFIA transcription factor with a decrease in GR expression as an outcome. McGowan et al (31) studied hippocampal samples obtained post-mortem from suicide victims with a history of childhood abuse. The authors observed increased methylation in the promoter of neuron-specific glucocorticoid receptor (NR3C1) and decreased mRNA levels compared to controls. No differences in glucocorticoid receptor expression were observed between suicide victims without a history of childhood abuse and controls. Oberlander et al (32) demonstrated in humans that the prenatal exposure to maternal depressed/ anxious mood is associated with the methylation status of the promoter and exon1F of the $\mathrm{NR} 3 \mathrm{C} 1$ gene in newborns.

It was reported that early-life stress in mice was associated with stable hypersecretion of corticosterone accompanied by a persistent increase in the expression of arginine vasopressin (AVP) in neurons of the hypothalamic paraventricular nucleus (33). The increase in AVP expression correlated with sustained DNA hypomethylation in $\mathrm{CpG}$ residues that serve as DNAbinding sites for the methyl CpG-binding protein 2 (MeCP2).

In another study (34), it was demonstrated that normal stress response in rats is dependent on the mother's affection during the early life of the child. The authors found that maternal care causes stable changes in promoter methylation of GR exon $1^{7}$. The offspring of "more affectionate" dams, measured by high pups licking and grooming, showed lower cytosine methylation level (site 16) and higher GR expression, allowing enhanced GR feedback sensitivity as compared to the offspring of "less affectionate" dams. The methylation differences were associated with altered histone acetylation and NGFI-A promoter binding and emerged during the very first week of pups' life.
The intergenerational transmission of psychological trauma has been studied in the offspring of Holocaust survivors (35). Holocaust exposure altered the methylation of FKBP5 gene, an important regulator of GR sensitivity, in exposed parents and their offspring. Methylation differences were found in the intron 7 glucocorticoid receptor binding sequence. Interestingly, in Holocaust survivors the methylation level was higher compared to controls, whereas in their offspring was lower. The observed altered methylation in parental exposure and offspring trauma exposure (childhood physical and sexual abuse) was differentially associated with sites in bins 3 and 2, respectively. Based on this, the authors suggested a possibility of site specificity in methylation depending on the environmental influences. Another example of intergenerational psychological trauma inheritance was provided by Vaage $e t$ al (36) who conducted a longitudinal study on Vietnamese refugees. The authors reported a high risk of mental disease in the third generation of refugees who were diagnosed with post-traumatic stress disorder on their arrival in Norway.

\section{THE EPIGENETIC DISEASE}

We can generally distinguish between two major roles of epigenetics: one in normal development, and the other in disease. While epigenetic changes are required for the regulation of the major biological processes including reprogramming during early embryogenesis, development and adaptation, they can be also correlated with some disease conditions. Epigenetic alterations can cause abnormal activation or silencing of genes and were described in many complicated disorders such as asthma, obesity, cardiovascular disease, lung disease, neurodegenerative and psychological disorders, addictions, and cancer.

Cancer was the first human disease that was related to epigenetics (37). As the maintenance of the normal methylation profile is of crucial importance for the precise gene expression and genome integrity, epigenetic alterations may lead to gene expression changes and may serve as a basis for carcinogenic development. In cancer cells, the normal activity of methyltransferase enzymes is disrupted (38) resulting in global hypomethylation (39) and local hypermethylation of normally unmethylated DNA regions (40). DNA hypomethylation can activate oncogenes and initiate chromosome instability, whereas DNA hypermethylation may provoke silencing of tumor suppressor genes. In addition to DNA methylation, histone modification patterns were also related to malignant diseases including breast and prostate cancer.

Some mental retardation disorders like Prader-Willi and 
Angelman syndromes, are other examples, where the disease phenotype was found to correlate with epigenetic changes (or "epimutations") leading to loss of genetic expression (41). A brain tissue obtained after the autopsy of a patient with autisms revealed a deficiency in MECP2 expression (42). A role of aberrant methylation in neurodegenerative diseases has also been suggested (43).

The unique features of epigenetic changes (such as reversibility, long-lasting effect, and renewability under endogenous or exogenous stimuli) may explain the differences between epigenetic and genetic diseases. These justify the variability, heterogeneity and individual course of most epigenetic diseases, a phenomenon, which could hardly be explained with genetic variations only. Moreover, taking into account the lack of known repair mechanisms regarding epimutations, we could expect an even greater environmental impact and a larger epigenetic component in the initiation and progression of the disease (44).

The available epigenetic data allow us to better understand the interplay between epigenetic modification, gene regulation, and diseases. The emerging role of epigenetics in disease may explain why many of the therapeutic approaches failed to reach the intended success in the past. A better understanding of epigenetic mechanisms involved in human disorders is expected to offer more effective therapies. The identification of disease-specific epigenetic marks may be helpful in developing diagnostic tools and the design of epigenetic drugs for targeted treatment. Potential sources for drug development based on epigenetics are DNA methylation inhibitors and histone deacetylase inhibitors.

Considering the reversible nature of epigenetic changes and the huge impact of the environment on the genome, another possibility emerges of regaining health and healing - through intended and targeted change in the environment. The study of environmentally induced epigenetic plasticity and the establishment of specific stimulus-epigenome correlations may potentially allow somewhat to purposely guide our epigenetic marks shaping the epigenome through a conscious choice of our lifestyle and living context.

\section{CONCLUSION}

In conclusion, some aspects we currently learned from the epigenetic revolution are that:

- Heredity is determined not only by the primary DNA sequence but also by epigenetic factors, which modulate gene expression
- Though conservative, the genome is dynamic and changeable in response to environmental conditions

- Nature and nurture are not separate units but have a combined influence mediated through epigenetics

- There are mechanisms for the epigenetic transmission of environmentally induced changes that occur long before conception from a parent to his offspring

- Emotional stress may cause epigenetic changes in our heredity molecules

- Epigenetic changes are related to many human disorders, providing possibilities for the development of more effective therapeutic approaches.

\section{CONFLICT OF INTEREST}

The author has no conflict of interest to disclose.

\section{ACKNOWLEDGEMENTS}

The author is very grateful to Dr. Georgi N. Bonchev for the critical reading of this manuscript and his beneficial remarks, and to the National Science Fund of Bulgaria, Grant KP$06-\mathrm{H} 25 / 5$ for the financial support of studies in epigenetics.

\section{REFERENCES}

1. Lander ES, Linton LM, Birren B, Nusbaum C, Zody $\mathrm{MC}$, Baldwin J, et al. Initial sequencing and analysis of the human genome. Nature 2001; 409: 860-921. DOI: $10.1038 / 35057062$

2. Orgel LE, Crick FH. Selfish DNA: the ultimate parasite. Nature 1980; 284: 604-607. DOI:10.1038/284604a0

3. de Koning AP, Gu W, Castoe TA, Batzer MA, Pollock DD. Repetitive elements may comprise over two-thirds of the human genome. PLoS Genet 2011; 7, e1002384. DOI:10.1371/journal.pgen.1002384

4. Gall JG. Chromosome structure and the $\mathrm{C}$-value paradox. J Cell Biol 1981; 91: 3-14. DOI:10.1083/jcb.91.3.3s

5. Waddington C H. The epigenotype. Endeavour 1942; 1: $18-20$

6. Wu C-t, Morris JR. Genes, genetics, and epigenetics: A correspondence. Science 2001; 293: 1103-1105. DOI:10.1126/science.293.5532.1103

7. Slotkin RK, Martienssen R. Transposable elements and the epigenetic regulation of the genome. Nat Rev Genet 2007; 8: 272-285. DOI:10.1038/nrg2072

8. Carey N. The Epigenetic Revolution. How Modern Biology is Rewriting our Understanding of Genetics, Disease and Inheritance. Columbia University Press, New York, 2012. 
9. Feil R, Fraga MF. Epigenetics and the environment: emerging patterns and implications. Nat Rev Genet 2012; 13: 97-109. DOI:10.1038/nrg3142

10. Baccarelli A, Bollati V. Epigenetics and environmental chemicals. Curr Opin Pediatr 2009; 21, 243-251. DOI:10.1097/mop.0b013e32832925cc

11. Barouki R, Gluckman PD, Grandjean P, Hanson M, Heindel JJ. Developmental origins of noncommunicable disease: implications for research and public health. Environ Health 2012; 11: 42. DOI: 10.1186/1476-069X-11-42

12. Tobi EW, Lumey LH, Talens RP, Kremer D, Putter H, Stein $\mathrm{AD}$, et al. DNA methylation differences after exposure to prenatal famine are common and timing- and sex-specific. Hum Mol Genet 2009; 18, 21: 4046-4053. DOI: $10.1093 / \mathrm{hmg} / \mathrm{ddp} 353$

13. Heijmans BT, Tobi EW, Stein AD, Putter H, Blauw GJ, Susser ES, et al. Persistent epigenetic differences associated with prenatal exposure to famine in humans. Proc Natl Acad Sci USA 2008; 105: 17046-17049. DOI: 10.1073/pnas.0806560105

14. Carito V, Parlapiano G, Rasio D, Paparella R, Paolucci $\mathrm{V}$, Ferraguti G, et al. Fetal alcohol spectrum disorders in pediatrics FASD and the paediatrician. Biomed Rev 2018; 29: 27-35. DOI: $10.14748 /$ bmr.v29.5847

15. Ciafrè S, Carito V, Tirassa P, Ferraguti G, Attilia ML, Ciolli $\mathrm{P}$, et al. Ethanol consumption and innate neuroimmunity. Biomed Rev 2017; 28: 49-61. DOI: 10.14748/ bmr.v28.4451

16. Ciafrè $\mathrm{S}$, Carito V, Ferraguti G, Greco A, Chaldakov G, Fiore $\mathrm{M}$, et al. How alcohol drinking affects our genes: an epigenetic point of view. Biochem Cell Biol 2018; 97 , 4: 345-356. DOI: $10.1139 / \mathrm{bcb}-2018-0248$

17. Ciafrè S, Ferraguti G, Greco A, Polimeni A, Ralli M, Ceci FM, et al. Alcohol as an early life stressor: Epigenetics, metabolic, neuroendocrine and neurobehavioral implications. Neurosci Biobehav Rev 2020; 118: 654-668. DOI: 10.1016/j.neubiorev.2020.08.018

18. Carito V, Ceccanti M, Ferraguti G, Coccurello R, Ciafrè $\mathrm{S}$, Tirassa $\mathrm{P}$, et al. NGF and BDNF alterations by prenatal alcohol exposure. Curr Neuropharmacol 2019; 17, 4: 308-317. DOI: 10.2174/1570159X15666170825101308

19. Ceccanti M, Coccurello R, Carito V, Ciafrè S, Ferraguti $\mathrm{G}$, Giacovazzo G, et al. Paternal alcohol exposure in mice alters brain NGF and BDNF and increases ethanol-elicited preference in male offspring. Addict Biol 2016; 21, 4: 776-787. DOI: $10.1111 / \mathrm{adb} .12255$
20. Tippin B, Pham P, Goodman MF. Error-prone replication for better or worse. Trends Microbiol 2004; 12, 6: 288295. DOI:10.1016/j.tim.2004.04.004

21. Gillespie MN, Wilson GL. Bending and breaking the code: dynamic changes in promoter integrity may underlie a new mechanism regulating gene expression. Am J Physiol Lung Cell Mol Physiol 2007; 292: L1-L3. DOI:10.1152/ ajplung.00275.2006

22. Ollikainen M, Smith KR, Joo EJ, Ng HK, Andronikos R, Novakovic $\mathrm{B}$, et al. DNA methylation analysis of multiple tissues from newborn twins reveals both genetic and intrauterine components to variation in the human neonatal epigenome. Hum Mol Genet 2010; 19, 21, 4176-4188. DOI: $10.1093 / \mathrm{hmg} / \mathrm{ddq} 336$

23. Waterland RA, Jirtle RL. Transposable elements: targets for early nutritional effects on epigenetic gene regulation. Mol Cell Biol 2003; 23: 15: 5293-5300. DOI:10.1128/ mcb.23.15.5293-5300.2003

24. Guy J, Gan J, Selfridge J, Cobb S, Bird A. Reversal of neurological effects in a mouse model of Rett syndrome. Science 2007; 315: 1143-1147 DOI:10.1126/science.1138389

25. Dias BG, Ressler KJ. Parental olfactory experience influences behavior and neural structure in subsequent generations. Nat Neurosci 2014; 17: 89-96. DOI: 10.1038/nn.3594

26. Dunn GA, Bale TL. Maternal high-fat diet promotes body length increases and insulin insensitivity in secondgeneration mice. Endocrinology 2009; 150: 4999-5009. DOI: 10.1210/en.2009-0500

27. Crews D, Gillette R, Scarpino SV, Manikkam M, Savenkova MI, Skinner MK. Epigenetic transgenerational inheritance of altered stress responses. Proc Natl Acad Sci USA 2012; 109, 23: 9143-9148. DOI: 10.1073/ pnas. 1118514109

28. Painter R, Osmond C, Gluckman P, Hanson M, Phillips $\mathrm{D}$, Roseboom T. Transgenerational effects of prenatal exposure to the Dutch famine on neonatal adiposity and health in later life. BJOG 2008; 115: 1243-1249. DOI: 10.1111/j.1471-0528.2008.01822.x

29. Veenendaal M, Painter R, de Rooij S, Bossuyt P, van der Post J, Gluckman P, et al. Transgenerational effects of prenatal exposure to the 1944-45 Dutch famine. BJOG 2013; 120: 548-554. DOI: 10.1111/1471-0528.12136

30. Moore R, Kaletsky R, Murphy C. Piwi/PRG-1 Argonaute and TGF- $\square$ mediate transgenerational learned pathogenic avoidance. Cell 2019; 177: 1827-1841. DOI: 10.1016/j. cell.2019.05.024 
31. McGowan PO, Sasaki A, D'Alessio AC, Dymov S, Labonté B, Szyf M, et al. Epigenetic regulation of the glucocorticoid receptor in human brain associates with childhood abuse. Nat Neurosci 2009; 12: 3: 342-348. DOI: $10.1038 / \mathrm{nn} .2270$

32. Oberlander TF, Weinberg J, Papsdorf M, Grunau R, Misri S, Devlin AM. Prenatal exposure to maternal depression, neonatal methylation of human glucocorticoid receptor gene (NR3C1) and infant cortisol stress responses. Epigenetics 2008; 2: 97-106. DOI:10.4161/epi.3.2.6034

33. Murgatroyd C, Patchev AV, Wu Y, Micale V, Bockmühl $\mathrm{Y}$, Fischer D, et al. Dynamic DNA methylation programs persistent adverse effects of early-life stress. Nat Neurosci 2009; 12: 1559-1566. DOI: 10.1038/nn.2436

34. Weaver I, Cervoni N, Champagne F, D’Alessio A, Sharma $\mathrm{S}$, Seckl J, et al. Epigenetic programming by maternal behaviour. Nat Neurosci 2004; 7: 847-854 DOI:10.1038/ nn1276

35. Yehuda R, Daskalakis NP, Bierer LM, Bader HN, Klengel $\mathrm{T}$, Holsboer F, et al. Holocaust Exposure Induced Intergenerational Effects on FKBP5 Methylation. Biol Psychiatry 2016; 80: 372-380. DOI: 10.1016/j.biopsych.2015.08.005

36. Vaage AB, Thomsen PH, Rousseau C, Wentzel-Larsen T, Ta TV, Hauff E. Paternal predictors of the mental health of children of Vietnamese refugees. Child Adolesc Psychiatry Ment Health 2011 ; 5: 2. DOI: 10.1186/1753-2000-5-2

37. Feinberg AP, Vogelstein B. Hypomethylation distinguishes genes of some human cancers from their normal coun- terparts. Nature 1983; 301: 89-92. DOI:10.1038/301089a0

38. Yokochi T, Robertson KD. Preferential methylation of unmethylated DNA by mammalian de novo DNA methyltransferase Dnmt3a. J Biol Chem 2002; 277: 11735-11745. DOI:10.1074/jbc.M106590200

39. Esteller M, Fraga MF, Guo M, Garcia-Foncillas J, Hedenfalk I, Godwin AK. DNA methylation patterns in hereditary human cancers mimic sporadic tumorigenesis. Hum Mol Genet 2001; 10: 3001-3007. DOI:10.1093/ $\mathrm{hmg} / 10.26 .3001$

40. Baylin SB, Herman JG. DNA hypermethylation in tumorigenesis: epigenetics joins genetics. Trends Genet 2000; 16: 168-174. DOI:10.1016/s0168-9525(99)01971-x

41. Egger G, Liang G, Aparicio A, Jones PA. Epigenetics in human disease and prospects for epigenetic therapy. Nature 2004; 429: 457-463. DOI:10.1038/nature02625

42. Nagarajan RP, Hogart AR, Gwye Y, Martin MR, LaSalle JM. Reduced MeCP2 expression is frequent in autism frontal cortex and correlates with aberrant MECP2 promoter methylation. Epigenetics 2006; 1: 4, e1-11. DOI:10.4161/epi.1.4.3514

43. Lu H, Liu X, Deng Y, Qing H. DNA methylation, a hand behind neurodegenerative diseases. Front Aging Neurosci 2013; 5: 1-16. DOI: 10.3389/fnagi.2013.00085

44. McCarrey JR. The epigenome as a target for heritable environmental disruptions of cellular function. Mol Cell Endocrinol 2012; 354, 1-2: 9-15. DOI: 10.1016/j. mce.2011.09.014 